\title{
Análise Hierárquica de Processos (AHP) aplicada à seleção de sistemas de revestimento de fachadas
}

Analytic Hierarchy Process (AHP) applied to the selection of facade cladding systems

Gustavo Macioski ${ }^{1}$ (b) orcid.org/0000-0001-8489-4328

Nicolle Talyta Arriagada Soto ${ }^{1}$ (D) orcid.org/0000-0003-2538-4744

Ludmila de Souza Freitas ${ }^{2}$ (D) orcid.org/0000-0003-1947-4196

Roberto Caldeira da Silva ${ }^{2}$ (D) orcid.org/0000-0002-7973-2663

Marcelo Henrique Farias de Medeiros ${ }^{1}$ (D) orcid.org/0000-0003-3112-9715

${ }_{1}^{1}$ Programa de Pós-Graduação em Engenharia de Construção Civil, Universidade Federal do Paraná, Curitiba-PR, Brasil

2 Programa de Pós-Graduação em Engenharia Civil, Universidade Tecnológica Federal do Paraná, Curitiba-PR, Brasil

E-mail do autor principal: Gustavo Macioski - gmacioski@gmail.com

\section{Resumo}

O aumento de novas tecnologias torna cada vez mais complexa a tomada de decisões na indústria da construção civil, seja para a especificação de materiais de construção ou para a adoção de métodos construtivos. Há, portanto, a necessidade de se estabelecer critérios objetivos que subsidiem a tomada de decisão quanto ao material mais apropriado a ser utilizado em uma construção. Desta forma, o objetivo deste trabalho é realizar uma análise comparativa de diferentes sistemas de revestimento de fachadas: revestimento argamassado, cerâmico, granito, vidro e ACM (Aluminum Composites Materials). Para isso foi aplicada a metodologia de análise hierárquica do processo (AHP) com base em parâmetros como: tempo, economia, ambiental, conforto, durabilidade e estético; sob três diferentes cenários. A partir dos resultados, verificou-se que o método se mostrou eficaz na tomada de decisão, sendo que as fachadas de argamassa e cerâmica ainda apresentam melhor relação custo/benefício em todos os cenários analisados. Constatou-se uma carência de valores de referências de propriedades de materiais de construção e sistemas construtivos; e observou-se como os parâmetros avaliados podem influenciar a decisão final da escolha do material. Assim, a AHP poderia ser utilizada em outros contextos de tomada de decisão na construção civil, desde que os dados dos parâmetros avaliados estejam disponíveis.

Palavras-Chave: Desempenho; Matriz de decisão; AHP; Sustentabilidade.

\section{Abstract}

The increase in new technologies makes decision making in the construction industry increasingly complex, whether for the specification of construction materials or the adoption of construction methods. Therefore, there is a need to establish objective criteria to support decision making for the most appropriate material to be used in a construction. Thus, the aim of this paper is to carry out a 
comparative analysis of different facade cladding systems: mortar, ceramic, granite, glass and ACM (Aluminum Composites Materials). For that, the methodology of Analytic Hierarchy Process (AHP) was applied based on parameters such as: time, economy, environment, comfort, durability and aesthetics; under three different scenarios. From the results, it was verified that the method proved to be effective in decision making, and the mortar and ceramic facades still present a better cost/benefit relation in all the analyzed scenarios. A lack of reference values of building material properties and building systems was found; and it was observed how the parameters evaluated can influence the final decision on the choice of material. Thus, AHP could be used in other decisionmaking contexts in construction, as long as the data of the assessed parameters are available.

Key-words: Performance; Decision matrix; AHP; Sustainability.

\section{Introdução}

A complexidade da tomada de decisão em projetos da construção civil é crescente conforme o aumento da disponibilidade de novos materiais no mercado, da introdução de novas tecnologias e programas de gestão da qualidade, assim como da exigência dos clientes e da crescente competitividade do setor. Desta forma, o tempo dispendido para se tomar uma decisão pode ser a diferença entre uma vantagem competitiva e a perda de mercado [1]. Para Medeiros, raramente a escolha é baseada em critérios técnicos confiáveis, sendo mais comum considerar somente aspectos estéticos (cor, textura, acabamento, etc.) e econômicos na seleção de materiais [2]. A construção civil ainda possui métodos arcaicos como preparo/mistura de materiais in loco sem controle tecnológico e uso de ferramentas improvisadas. Assim, é considerado um dos mercados mais difíceis para adaptação de novas tecnologias [3]. Há, portanto, a necessidade de se estabelecer critérios objetivos que subsidiem a tomada de decisão quanto ao material mais apropriado para determinada aplicação, levando-se em consideração outros parâmetros como a durabilidade, produtividade, conforto, manutenção e impacto ambiental da construção.

Assim, considerando-se a importância da tomada de decisão na escolha de materiais empregados em fachadas, o objetivo deste trabalho é realizar uma análise comparativa de diferentes sistemas de revestimento de fachadas de edificações: revestimento argamassado, revestimento cerâmico, revestimento em granito, vidro e ACM (Aluminium Composites Materials). Para isso foi aplicada a metodologia de análise hierárquica com base em parâmetros como: tempo, economia, agressividade ao meio ambiente, conforto, durabilidade e estético; sob três diferentes pontos de vista: Engenheiros, Arquitetos e Consumidores. Neste sentido, este trabalho visa contribuir para a substituição das decisões de caráter subjetivo e evidenciar, dessa forma, os aspectos técnicos e objetivos para a análise e decisão entre esses cinco tipos de revestimento. $O$ uso desta ferramenta para seleção de materiais já foi publicado por Mattana et al. [4], porém, com um enfoque diferente, pois nesta pesquisa não foi avaliado o aspecto ambiental e econômico e as opções de escolha eram as proporções de mistura de argamassas de revestimento apenas, diferente do trabalho atual que compara opções de sistemas de revestimentos adicionais considerando aspectos abrangentes como sustentabilidade, durabilidade e conforto (não considerados no estudo anterior).

\section{Aspectos de tomada de decisão}

São diversos os parâmetros que podem ser considerados para uma tomada de decisão quanto à seleção de materiais e seu uso. Nesta seção serão tratados os principais aspectos relacionados a sistemas de fachadas que devem ser considerados no processo de escolha do sistema construtivo.

\subsection{Economia e produtividade}

O custo é um dos fatores de grande importância na especificação dos materiais [5]. Além de se analisar o custo inicial de implantação do material, as metodologias mais recentes analisam também o custo de manutenção ao longo do tempo, de forma a avaliar o custo global. As degradações identificadas na edificação ou manifestações patológicas podem se desenvolver em todos e quaisquer elementos das edificações [6-7], porém, do ponto de vista da manutenção do sistema, os revestimentos de fachada 
exigem cuidados por se encontrarem em condições desfavoráveis de exposição, como incidência de chuva, sol e vento [2].

Outro parâmetro que pode influenciar uma tomada de decisão é a produtividade, que está relacionada com o desempenho da mão-de-obra envolvida. A melhor capacitação e correta definição das técnicas a serem empregadas resultam em um aumento da produtividade e consequentemente a diminuição dos custos com perdas de material, manutenções e atrasos de cronograma [5].

\subsection{Sustentabilidade}

Os indicadores de sustentabilidade são aqueles que, baseados em parâmetros específicos, informam o grau de sustentabilidade de algo, para que a tomada de decisão no sentido de construções mais sustentáveis possa ser feita [8]. Dentre os diversos meios para avaliar se um material ou componente é mais ou menos sustentável atualmente se tem dado especial atenção para a energia embutida e emissão de $\mathrm{CO}_{2}$ [9], ainda que existam inúmeras propriedades a serem avaliadas, inclusive seus efeitos do ponto de vista de poluição e contaminação (radiação, elementos pesados e partículas finas) presente em diversos materiais de construção [10].

Além da relevância da energia operacional na avaliação dos impactos ambientais, a qual é demandada pelo uso de equipamentos ao longo da vida útil da edificação, a determinação da energia embutida inicial também se faz necessária [9]. A energia embutida é definida como o total de insumos energéticos, tanto diretos como indiretos, necessários para a fabricação e distribuição de um produto em todas as etapas pré-operacionais de seu ciclo de vida [11-12].

Os dados necessários para a estimativa da energia embutida de um material podem ser obtidos através de métodos analíticos (percorrendo o processo de produção, desde a extração dos materiais brutos até o produto final) ou de métodos estatísticos (examinando uma indústria completa em um dado país, obtendo o seu uso total de energia e dividindo-o pela produção no mesmo período) [13-14].

Nas décadas de 1970 e 80, a preocupação ambiental da sociedade estava focada nos processos de produção, assim, os regulamentos ambientais concentraram-se na poluição das indústrias. No entanto, no final dos anos 1980 e durante a década de 90 , foi cada vez mais reconhecido que tanto a fase de utilização quanto o processo de descarte são importantes no ciclo de vida do produto. Deste modo, esse cenário exigiu uma nova abordagem para a concepção dos produtos, visando abranger todas as fases do ciclo de vida e o desenvolvimento sustentável. Para o setor da construção civil, isso significa que é recomendável produzir estruturas com um mínimo impacto ambiental, sobre todas as fases do ciclo de vida da edificação, visando minimizar o consumo de energia de operação e otimizar a utilização de materiais de construção [15].

De acordo com Tello e Ribeiro, a cadeia produtiva da construção civil tem impactos ambientais difusos e de longo prazo, com muitas particularidades locais, no entanto os resultados apresentados sempre se mostram significativos em escala global [11]. Assim, o setor da construção é responsável por $12 \%$ do consumo total de água, $40 \%$ de todos os resíduos gerados pela sociedade, e a produção de cimento é responsável por $5 \%$ das emissões de gases de efeito estufa [16]. Ainda que resíduos possam ser reutilizados na produção de novos materiais, é impossível neutralizar o impacto causado pela indústria da construção civil durante a extração, fabricação e aplicação de materiais de construção [17].

Estes e outros fatores ambientais tem motivado um número crescente de projetistas, construtores e usuários na busca por estratégias ambientalmente mais sustentáveis de projeto e construção. No entanto, em comparação com outros "produtos", edifícios são mais difíceis de serem avaliados, pelos seguintes motivos: são grandes em escala, complexos em materiais e funções, e temporalmente dinâmicos devido à vida útil limitada de componentes de construção e alterações das necessidades dos usuários. Além disso, seu processo de produção é menos padronizado em função do caráter único de cada edifício.

Também pode-se afirmar que existem informações quantitativas limitadas sobre os impactos ambientais, da produção e fabricação de materiais de construção, ou o próprio processo de construção e demolição [18].

\subsection{Conforto e desempenho}

Para Durmisevic e Iersel [19], projetos precisam considerar mudanças na economia e estilo de vida da

DOI: $10.25286 /$ repa.v6i1.1381 
população, e permitir adaptações e renovações no ambiente construído. Esse contexto possibilita influenciar consideravelmente a maneira de projetar e construir no futuro. Babik e Torres [20] destacam que a arquitetura tem relação direta com o desempenho de seus usuários no desenvolvimento de suas atividades, pois oferecer um espaço adequado com bons índices de iluminação, ventilação e conforto térmico é essencial e de extrema importância.

Visando atender ao consumo e às novas demandas que surgem pelo modo de vida da sociedade contemporânea, a construção civil necessita explorar, cada vez mais, uma quantidade expressiva de materiais. Com isso, a prática da especificação de materiais tende a se tornar cada vez mais exigente, tendo em vista os critérios de seleção tradicionais, tais como a estética, o custo, o potencial para se construir em curto prazo, além das preocupações sociais [21]. Abeysundara et al. [22] classificam como preocupações sociais itens como conforto térmico e acústico, boa qualidade do ar interior e boa qualidade estética, ou seja, aspectos que envolvam benefícios às percepções e ao conforto humano.

Segundo OLIVEIRA et al. [8], a influência de ações como as altas temperaturas, umidade, radiação solar, etc., é particularmente significativa nos componentes localizados na envoltória (coberturas e fachadas) dos edifícios, por estarem continuamente submetidos ao aquecimento pelos raios solares e, do mesmo modo, ao resfriamento em razão da ocorrência de chuvas ou de alterações de temperatura do ambiente. Portanto, tanto a durabilidade física dos componentes de fachadas de edifícios, quanto os níveis de conforto ambiental e, consequentemente, de sustentabilidade apresentados por uma edificação, estão relacionados com a composição dos materiais empregados na fachada.

Neste sentido, é possível afirmar que o consumo energético das edificações depende dos ganhos ou perdas de calor pela envoltória da edificação, que, associados à carga interna gerada pela sua fase operacional, resultam no consumo por sistemas de climatização, iluminação, entre outros equipamentos [23].

A contemplação de conceitos que visam à maior sustentabilidade no projeto e, por conseguinte, na tomada de decisão de métodos construtivos, induz à afirmativa de que é necessário desenvolver novas técnicas, ferramentas e metodologias que colaborem com os novos compromissos a serem cumpridos e que sejam direcionadas aos profissionais para que possam ser inseridas na prática da atividade profissional [24].

É possível afirmar que, ainda nos dias atuais, é incipiente e pouco abrangente a atuação dos projetistas na adoção de parâmetros denominados como sustentáveis na decisão por materiais e métodos construtivos. Esta lacuna vem acompanhada da dificuldade na contemplação de princípios de sustentabilidade no processo de elaboração do projeto, sendo possível mencionar as seguintes causas: ausência de critérios sistematizados para auxiliar nas escolhas; resistência e falta de conhecimento do cliente pelo custo de investimento em técnicas e materiais diferenciados; resistência por parte dos construtores e incorporadores; falta de divulgação e normatização de determinadas tecnologias e soluções; ausência de informações sobre o tema, entre outros [25-26].

\section{Metodologia}

Nesta seção estão apresentados os sistemas de fachadas analisados na pesquisa, os dados considerados nos cálculos, e será descrito o processo de análise aplicado. Os cálculos detalhados e as composições montadas são apresentadas como documento suplementar.

\subsection{Sistemas de fachada}

Foram considerados os principais sistemas de revestimento de fachadas utilizados atualmente no Brasil: argamassa pintada, cerâmica, granito, vidro e ACM; conforme apresentado na Figura 1. O memorial descritivo dos sistemas (materiais, camadas e acabamentos) é apresentado de forma detalhada nos documentos complementares.

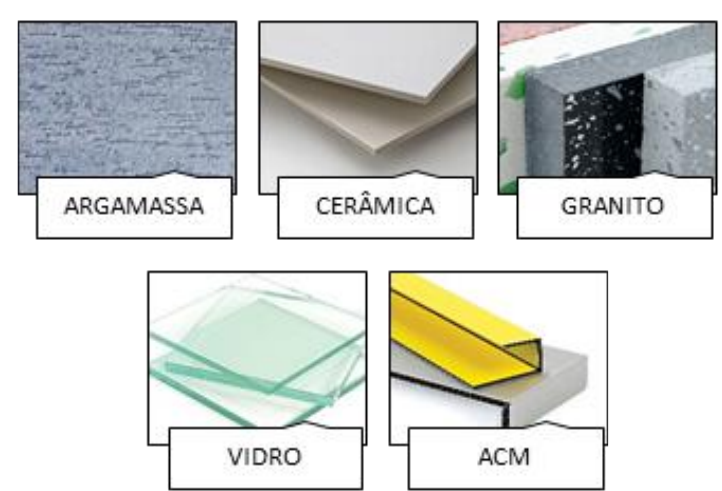

Figura 1: Tipos de revestimentos de fachada avaliados. 


\subsection{Cenários e aspectos considerados}

O desenvolvimento da metodologia de tomada de decisão levou em consideração diferentes cenários, onde cada um dos parâmetros poderia ser mais ou menos influente, conforme metodologias propostas por Medeiros [2] e por Kondo [5]. Os cenários (pontos de vista de decisores) são: Engenheiros, Arquitetos e Consumidores; e levaram em consideração a visão que estes agentes possuem sobre os aspectos relevantes na escolha da fachada.

A Figura 2 apresenta os parâmetros considerados em relação ao: tempo, economia, meio ambiente, conforto, durabilidade e estético; nos diferentes cenários de decisores.

A opção de avaliar os sistemas sob esses pontos de vista surgiu da necessidade de entender como profissionais da indústria da construção civil, que atuam no projeto e na execução de obras, priorizam diferentes aspectos na determinação do melhor custo/benefício de um sistema de fachada.

Os dados utilizados para os cálculos foram obtidos por meio da elaboração de composições por metro quadrado para cada fachada e as fontes utilizadas para a obtenção dos valores são apresentadas nos documentos complementares.

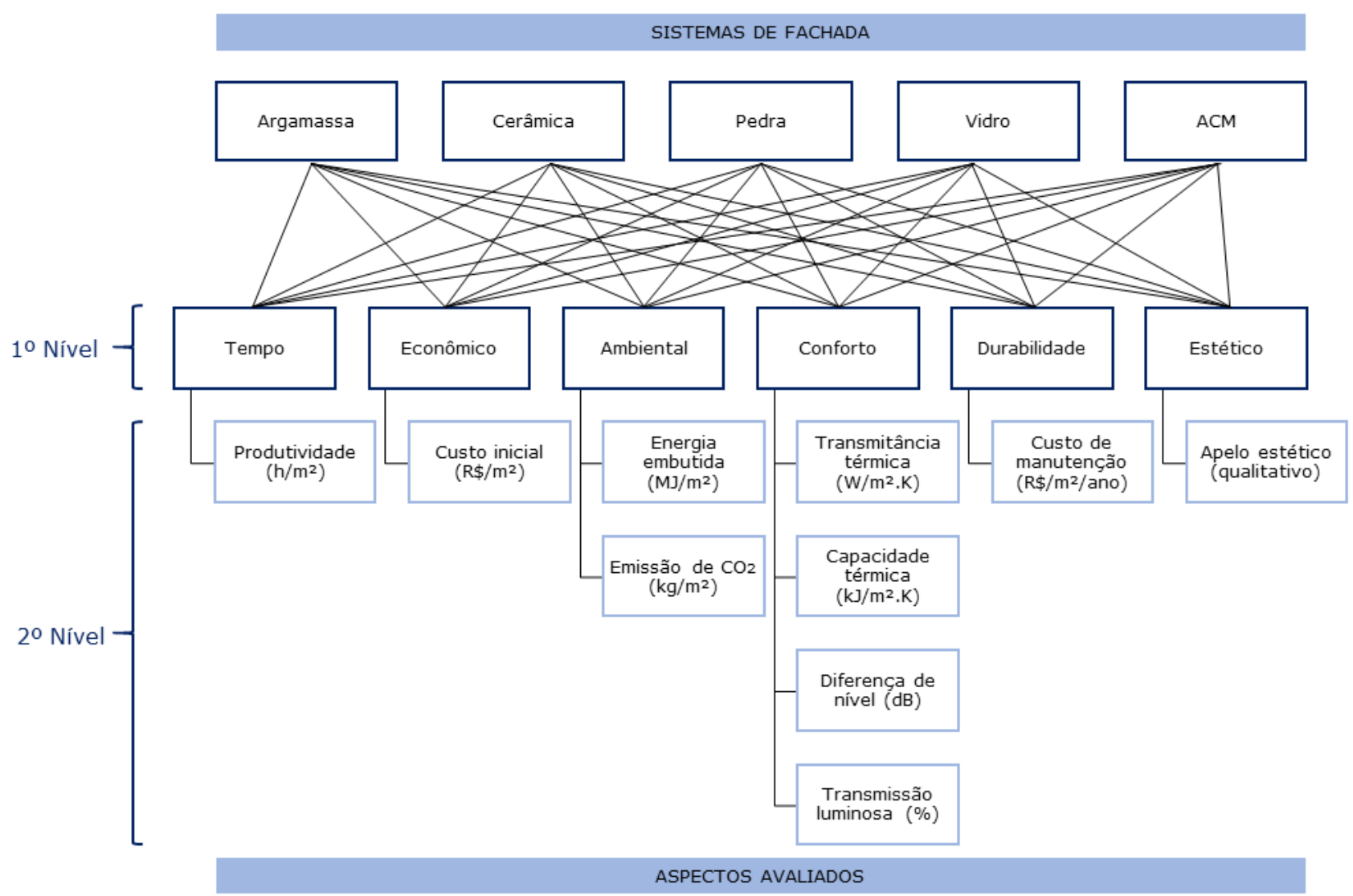

Figura 2: Níveis hierárquicos avaliados.

\subsection{Aplicação da análise hierárquica}

A aplicação da análise hierárquica do processo (AHP) seguiu as prescrições da ASTM E 1765 [27]. O método se destina a tomada de decisões complexas [28], e pode ser utilizada para diversos setores. Como previsto pela normativa, os processos foram:

- Identificar das alternativas e atributos;
- Estabelecer significância relativa entre pares de atributos;

- Avaliação da consistência das respostas;

- Elaborar matriz de decisão;

- Comparação e escolha das alternativas.

A identificação das alternativas e dos atributos foi apresentada na Figura 2, estabelecendo-se níveis 
hierárquicos às propriedades dos sistemas de fachada escolhidos. Para se estabelecer a significância relativa entre os atributos, foram aplicados questionários em um escritório da cidade de Curitiba-PR no ano de 2018 com os profissionais da construção civil (engenheiros e arquitetos) e clientes de modo a avaliar de forma quantitativa a relação entre os aspectos considerados.

Espera-se que os profissionais da construção civil (engenheiros e arquitetos) possuam uma visão técnica padronizada sobre as propriedades dos materiais, contudo, a percepção dos consumidores pode ser afetada por aspectos culturais e socioeconômicos dos entrevistados.

O preenchimento do questionário foi realizado de forma conjunta e simultânea, em grupos de 5 pessoas. O critério de apelo estético dos sistemas de fachadas também foi definido por cada um dos decisores durante a aplicação dos questionários. Para - estabelecimento da significância relativa dos atributos foi considerada a escala de comparação proposta pela ASTM E 1765 [27], como apresentado na Tabela 1.

Tabela 1: Escalas de comparação da ASTM E 1765 [27]

\begin{tabular}{cc}
\hline Comparação & Escala \\
\hline A tem igual importância que B & 1 \\
\hline A levemente mais importante que B & 3 \\
\hline A mais importante que B & 5 \\
\hline A muito mais importante que B & 7 \\
\hline A extremamente mais importante que B & 9 \\
\hline
\end{tabular}

profissionais, inconsistências podem ocorrer principalmente quando existir um grande número de julgamentos [29].

Após a aplicação do questionário, para a avaliação da coerência nas respostas dos questionários, foi calculada a razão de consistência (RC) que pode ser definida como uma razão entre 0 índice de consistência (IC) e o índice de consistência randômico (IR) [29-30].

\section{Resultados e discussão}

Após elaborada a composição por metro quadrado de cada um dos sistemas de fachada, considerando suas camadas conforme especificado pelo memorial descritivo, foi possível obter os valores apresentados na Tabela 2.

Durante a cálculo notou-se grande variação nos valores apresentados para as propriedades ambientais e de conforto. Em parte, pelos autores utilizarem metodologias diferentes para a determinação destes parâmetros; ou pela dificuldade de análise de materiais em conjunto, uma vez que o cálculo da propriedade total do sistema de fachada exige a aplicação de modelos e metodologias ainda pouco exploradas.

Mesmo quando os julgamentos paritários estão fundamentados na experiência e conhecimento de Tabela 2: Propriedades das fachadas avaliadas

\begin{tabular}{|c|c|c|c|c|c|}
\hline Propriedade & Argamassa & Cerâmica & Granito & Vidro & ACM \\
\hline Produtividade $\left(\mathrm{h} / \mathrm{m}^{2}\right)$ & 6,17 & 4,66 & 4,36 & 6,30 & 2,80 \\
\hline Custo inicial $\left(\mathrm{R} \$ / \mathrm{m}^{2}\right)$ & 94,05 & 248,09 & 309,90 & 1374,52 & 308,85 \\
\hline Energia embutida $\left(\mathrm{MJ} / \mathrm{m}^{2}\right)$ & 128,45 & 113,46 & 225,18 & 524 & 171,5 \\
\hline Emissão de $\mathrm{CO} 2\left(\mathrm{~kg} / \mathrm{m}^{2}\right)$ & 10,46 & 4,5 & 17,31 & 12,49 & 11,52 \\
\hline Transmitância térmica $\left(\mathrm{W} / \mathrm{m}^{2} \cdot \mathrm{K}\right)$ & 2,46 & 3,72 & 2,36 & 5,48 & 11,25 \\
\hline Capacidade térmica $\left(\mathrm{kJ} / \mathrm{m}^{2} \cdot \mathrm{K}\right)$ & 145 & 100 & 210 & 7 & 7 \\
\hline Diferença de nível à $1000 \mathrm{~Hz}(\mathrm{~dB})$ & 0,023 & 0,043 & 0,035 & 0,015 & 0,09 \\
\hline Iluminância geral (lux) & $0 \%$ & $0 \%$ & $0 \%$ & $14 \%$ & $0 \%$ \\
\hline Custo de manutenção $\left(\mathrm{R} \$ / \mathrm{m}^{2}\right)$ & 2,83 & 2,2 & 7,27 & 12,65 & 12,65 \\
\hline Apelo estético & \multicolumn{5}{|c|}{ Determinada por questionário para cada cenário } \\
\hline
\end{tabular}

Neste sentido, a criação de bancos de dados com todas as propriedades dos materiais é essencial para a correta aplicação de metodologias como a AHP, e a incorporação destas propriedades em softwares de modelagem da informação da construção (Building Information Modeling, BIM) poderia auxiliar na 39 tomada de decisão ao final do projeto. Para isso é necessária uma padronização nos métodos de obtenção e incorporação nos modelos.

Contudo, o levantamento a partir de consulta aos fornecedores revela que os dados, na maioria dos 
casos, não são disponibilizados. Além disso, nenhuma mídia disponibiliza a totalidade das informações necessárias ao projeto, sendo apontada a existência de dificuldades na obtenção de informações em geral. Assim, para a correta aplicação da AHP, é necessária a busca cuidadosa de parâmetros em diferentes fontes, de forma a garantir que a mesma metodologia foi utilizada, tornando os dados equivalentes e aptos para aplicação no método de tomada de decisão.

Após aplicação dos questionários, foi possível avaliar quais parâmetros apresentam maior grau de importância na decisão dos cenários decisores, como apresentado na Figura 3.

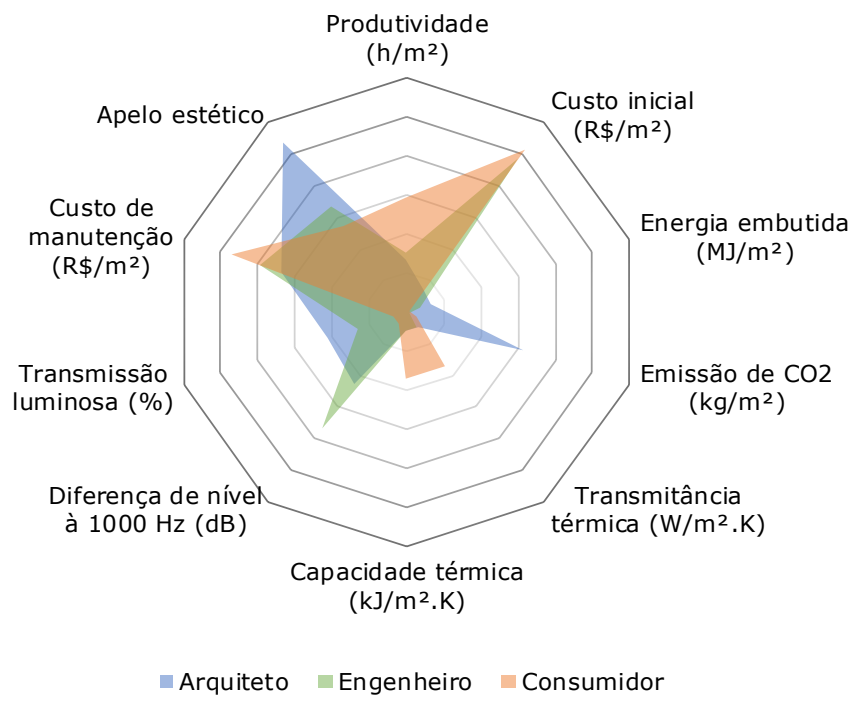

Figura 3: Grau de importância dos parâmetros avaliados.

Da análise da Figura 3, de uma forma geral é possível observar uma baixa preocupação, nos três cenários avaliados, com aspectos ambientais - energia embutida e emissão de $\mathrm{CO}_{2}$; ao passo que o apelo estético demostra impacto para todos os grupos. Pode-se notar, também, a diferente percepção que cada grupo estudado tem sobre as propriedades de cada sistema de fachada: segundo dados coletados com arquitetos, itens mais relevantes são aqueles ligados aos aspectos de conforto, economia e estética. Enquanto que os engenheiros atribuem mais relevância aos aspectos econômicos e de durabilidade. Na visão dos consumidores, por sua vez, os custos (inicial e de manutenção) são de extrema relevância, juntamente com o aspecto estético.

Após a compilação das respostas dos questionários, foi realizado o teste de consistência de modo a verificar a coerência nas respostas obtidas, bem como, se a comparação dos critérios estabelecidos é proporcional. Na Tabela 3 são apresentados os resultados obtidos.

Tabela 3: Teste de inconsistência

\begin{tabular}{c|c|c|c}
\hline & Engenheiro & Arquiteto & Consumidor \\
\hline $\begin{array}{c}\text { Razão de } \\
\text { consistência (RC) }\end{array}$ & $26 \%$ & $10 \%$ & $76 \%$ \\
\hline
\end{tabular}

Matrizes consistentes devem apresentar razões (RC) inferiores à $10 \%$, contudo, a partir dos resultados obtidos e da matriz utilizada, nota-se que em matrizes complexas o uso da escala proposta pela ASTM E 1765 [27] impede que o RC se encontre abaixo do limite proposto por Saaty [30]. Como o maior grau de comparação que pode ser utilizado é 9 , quando um parâmetro é de extrema importância comparado a outro, uma segunda comparação não pode apresentar um grau superior a primeira, devido a limitação na escala.

Mesmo após obtido o resultado da análise hierárquica, é importante a realização do teste de inconsistência para avaliar se a matriz utilizada (respostas dos decisores) são coerentes e, consequentemente, irão gerar uma resposta coerente. O aumento da inconsistência ocorre quando se aumentam os números de variáveis, quando a escala de comparação é reduzida ou quando o decisor se contradiz durante o questionário.

Avaliando-se os resultados obtidos na Tabela 3, é possível afirmar que os arquitetos demonstram uma maior coerência nas respostas quando comparado aos engenheiros, enquanto que os consumidores chegam a contradizer respostas dadas quando compiladas as comparações realizadas. Desta forma, um profissional da área técnica teria mais capacidade de tomar uma decisão relevante à escolha e ao projeto de uma fachada.

Diante dos aspectos mais relevantes para cada grupo e dos valores referentes a cada uma das características estudadas, foi calculado, então, o valor da decisão normalizada para cada grupo, como apresentado na Figura 4. 


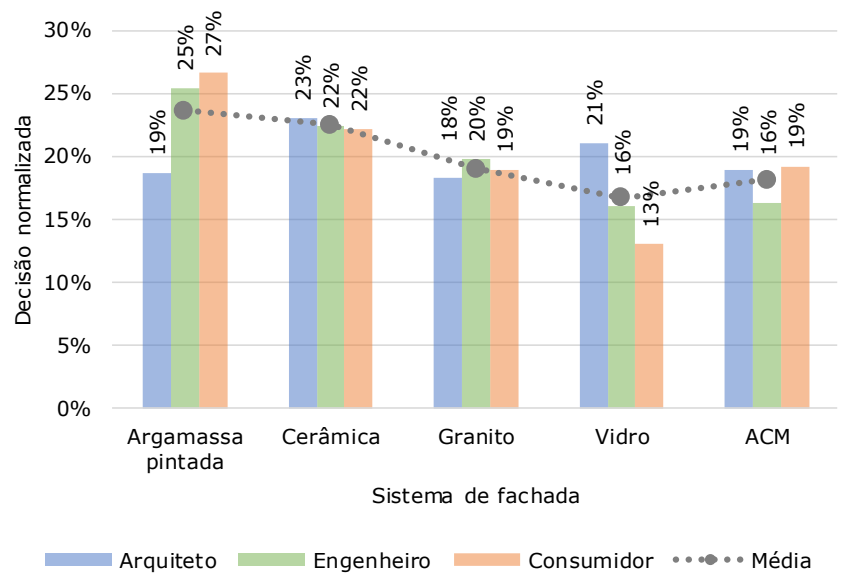

Figura 4: Tomada de decisão nos cenários

Como pode-se observar na Figura 4, tanto para o grupo de engenheiros como para o grupo de consumidores, o sistema a ser escolhido seria o de argamassa pintada: esse é o sistema com menores custo inicial e de manutenção, uma vez que esses aspectos (econômico e de durabilidade) foram relevantes para esses grupos, o que influenciou diretamente nesse resultado. Em segunda e terceira posição de escolha encontram-se os sistemas de cerâmica e de granito, respectivamente.

Por outro lado, para o grupo de Arquitetos, o sistema de fachada a ser escolhido seria o cerâmico, influenciado principalmente pelos aspectos ambiental, econômico e de durabilidade (Figura 3), seguido pela fachada de vidro. Se consideradas as três decisões de forma simultânea como uma média dos três cenários, é possível observar que o sistema de fachada de argamassa pintada, de fato, apresenta o maior índice de decisão, como pode ser observado na Figura 5.

As fachadas que apresentaram a maior variação nos índices de decisão foram as de argamassa pintada e a de vidro, com desvio padrão de $4,3 \%$ e $4,1 \%$, respectivamente. A fachada de vidro apresentou 0 menor índice de decisão, enquanto os sistemas de argamassa pintada e de cerâmica se destacaram.

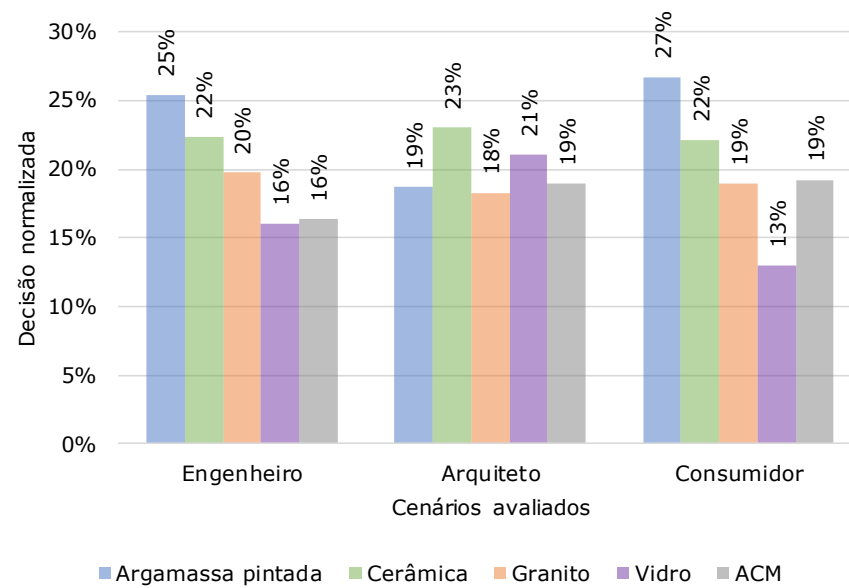

Figura 5: Comparação dos sistemas de fachada escolhidos

Ainda que aspectos ambientais e de conforto sejam cada vez mais influentes na tomada de decisão e detalhamento de projetos, o custo de materiais com melhor desempenho térmico, acústico e sustentável ainda é uma barreira para o desenvolvimento e implantação de novos sistemas construtivos e de tecnologias inovadoras no Brasil. A indústria da construção apresenta baixa produtividade e grande necessidade de aumento de sua eficiência e competitividade com uso de tecnologia da informação. A falta de informações nos processos da construção pode levar a decisões erradas ou provocar erros de execução e projeto, com consequente retrabalho [31].

O método de análise hierárquica (AHP) mostrou-se eficiente na tomada de decisão para escolha do sistema de fachada, e poderia ser replicado para diferentes sistemas construtivos e tipos de projeto. Observa-se que os maiores problemas desta metodologia estão ligados à disponibilidade de dados e ao grau de importância dada a cada parâmetro. Por este motivo, a simulação de diferentes cenários é essencial à análise posterior do resultado, de forma a validar a tomada de decisão feita.

\section{Conclusão}

Após análise comparativa de diferentes sistemas de revestimento de fachadas utilizando a metodologia de análise hierárquica foi possível concluir:

- Existe carência de valores de referências de propriedades de materiais e sistemas construtivos, bem como uma metodologia única para sua obtenção; 
- A escala de comparação utilizada pela ASTM E 1765 pode gerar inconsistência em matrizes com mais de 5 variáveis, contudo, a aplicação de questionários e análise de múltiplos cenários é essencial na tomada de decisão final;

- O método AHP se mostrou eficiente na tomada de decisão em sistemas complexos de forma rápida e objetiva;

- As fachadas de argamassa e cerâmica ainda apresentam melhor relação custo/benefício devido aos custos associados aos sistemas construtivos;

- Observou-se diferença de visão dos representantes dos três cenários quanto aos aspectos avaliados, porém ressalta-se que esta constatação é restrita aos atores usados nas entrevistas, não sendo um número relevante de representantes de cada cenário. Porém, trata-se de uma comparação interessante que poderia ser melhor investigada e validada em um estudo com uma maior amostra

\section{Referências}

[1] GONDIM, I. A. Modelo de apoio a decisão para seleção de tecnologias de revestimento de fachadas. Dissertação (Mestrado em Engenharia) Universidade Federal do Rio Grande do Sul. Porto Alegre, 2007.

[2] MEDEIROS, J. S. Tecnologia e projeto de revestimentos cerâmicos de fachadas de edifícios. Tese de doutorado. Escola Politécnica, Universidade de São Paulo. São Paulo, 1999.

[3] COSTELLA, M., CARUBiM, K., PAGLiARI, C., SOUZA, N. Avaliação da aplicação da norma de desempenho: estudo de caso em cinco empreendimentos. Revista de Engenharia Civil IMED, Porto Alegre, Vol. 4, n. 2, pag. 55-74, 2017.

[4] MATTANA, A. J.; MEDEIROS, M. H. F.; SILVA, N. G.; COSTA, M. R. M. M. Análise hierárquica para escolha entre agregado natural ou areia de britagem de rocha para confecção de argamassas de revestimento. Ambiente Construído (Online), v. 12 , p. 63-79, 2012.

[5] KONDO, S. T. Subsídios para seleção dos principais Revestimentos de Fachada de Edifícios. Monografia. Escola Politécnica da Universidade de São Paulo. Programa de Educação Continuada em Engenharia. 2003.

[6] RODRIGUES, A. O.; PALIGA, C. M.; TORRES, A. S. Análise da influência do horário do dia e da época do ano em imagens fotográficas para identificação de manifestações patológicas em fachadas na cidade de Pelotas/RS. Revista de Engenharia Civil IMED, Passo Fundo, v. 4, n. 1, p. 48-62, ago. 2017.

[7] KURZ, M., FERREIRA, C., RODRIGUES, A., SILVA, V., MEDVEDOVSKI, N., PALIGA, C., TORRES, A. Percepção do usuário em relação à presença de manifestações patológicas em fachadas: estudo de caso. Revista de Engenharia Civil IMED, v5 n1, 3-19, 2018.

[8] OLIVEIRA, C. N. O paradigma da sustentabilidade na seleção de materiais e componentes para edificações. Dissertação de mestrado. Universidade Federal De Santa Catarina. Florianópolis, 2009.

[9] JOHN, V. M.; OLIVEIRA, D. P.; AGOPYAN, V. Critérios de sustentabilidade para seleção de materiais e componentes-uma perspectiva de sustentabilidade para países em desenvolvimento. Journal of Building Environment, 2006.

[10] MACIOSKI, G.; SOTO, N. T. A.; MAZER, W.; CORREA, J. N. Avaliação dos níveis de concentração de radônio em marmorarias de Curitiba-PR. Revista De Geologia (Fortaleza), v. 30, p. 19-31, 2017.

[11] TELLO, R.; RIBEIRO, F. B. Guia CBIC de Boas Práticas em Sustentabilidade na Indústria da Construção. Nova Lima: Brasília: Câmara Brasileira da Indústria da Construção; Serviço Social da Indústria, 2012.

[12] TAVARES, S. F. Metodologia de Análise do Ciclo de Vida Energético de Edificações Residenciais Brasileiras. Tese de doutorado Programa de Pós-Graduação em Engenharia Civil Universidade Federal de Santa Catarina. Florianópolis, 2006.

[13] SZOKOLAY, S. V. The environmental imperative. International Conference in passive and low energy architecture - PLEA, v14, Kushiro: PLEA, 1997.

[14] MANFREDINI, C.; SATTLER, M. A. Estimativa da energia incorporada a materiais de cerâmica vermelha no Rio Grande do Sul. Ambiente Construído, v. 5, n. 1, p. 23-37, 2005.

[15] THORMARK, C. Recycling Potential and Design for Disassembly in Buildings. Doctoral Thesis Lund Institute of Technology - Lund University. Lund, Suécia, 2001. 
[16] PNUD - Programa das Nações Unidas para o Desenvolvimento, Objetivos do Desenvolvimento do Milênio. 2012.

[17] FROTTE, C.; NUBILA, C. S. A.; NAGALLI, A.; MAZER, W.; MACIOSKI, G.; OLIVEIRA, L. O. S. Estudo das propriedades físicas e mecânicas de concreto com substituição parcial de agregado natural por agregado reciclado proveniente de RCD. Materia-Rio de Janeiro, v. 22, p. e11811, 2017.

[18] SCHEUER, C., KEOLEIAN, G.A.; REPPE, P. Life cycle energy and environmental performance of a new university building: modeling challenges and design implications. School of Natural Resources and Environment. University of Michigan, 2003.

[19] DURMISEVIC, E., IERSEL, T. Life cycle coordination of materials and their functions at connections design for total service life of buildings and its materials. Proceedings of the 11 th Rinker International Conference, CIB Deconstruction and Materials Reuse, CIB Publication. Amsterdam, 2003.

[20] BABICK, C. S.; TORRES, M. C. A. Análise do desempenho térmico, lumínico e de ventilação natural de projeto padrão de edifício escolar do FNDE de acordo com os requisitos de normas e referenciais nacionais e internacionais nas 8 zonas bioclimáticas brasileiras. Revista de Engenharia Civil IMED, Passo Fundo, v. 4, n. 2, p. 110-128, dez. 2017

[21] HUANG, H.; ZHANG, L.; LIU, Z.; SUTHERLAND, J.W. Multicriteria decision making and uncertainty analysis for materials selection in environmentally conscious design. The International Journal of Advanced Manufacturing Technology, Volume 52, Issue 5-8, pp 421-432, 2011.

[22] ABEYSUNDARA, U. G. Y.; BABEL, S.; GHEEWALA, S. A matrix in life cycle perspective for selecting sustainable materials for buildings in Sri Lanka. Building and Environment, v. 44, n. 5, 2009.

[23] CARLO, J. C. Desenvolvimento de Metodologia de Avaliação da Eficiência Energética do Envoltório de Edificações Não-Residenciais. Tese (Doutorado em Engenharia Civil) Universidade Federal de Santa Catarina, Florianópolis, 2008.

[24] VAN CAUWENBERGH, N., BIALA, K., BIELDERS, C., BROUCKAERT, V., FRANCHOIS, L., CIDAD, V. G., SAUVENIER, X. Safe: a hierarchical framework for assessing the sustainability of agricultural systems. Agriculture, Ecosystems and Environment, 120(2-4):229-242, 2007.

[25] FAGUNDES, C.M.N. Contribuições para uma arquitetura mais sustentável. Salvador, BA. Dissertação Mestrado - Escola Politécnica Universidade Federal da Bahia. Salvador, 2009.

[26] MARTINEZ, L.D.; AMORIM, S.R.L. Inserção de aspectos sustentáveis no projeto de arquitetura unifamiliar e capacitação de profissionais de arquitetura em Niterói. Congresso nacional de excelência em gestão: energia, inovação, tecnologia e complexidade para a gestão sustentável, 6, Niterói, 2010.

[27] AMERICAN SOCIETY FOR TESTING AND MATERIALS. ASTM E 1765 - Standard Practice for Applying Analytical Hierarchy Process (AHP) to Multiattribute Decision Analysis of Investments Related to Buildings and Building Systems. West Conshohocken. 2002.

[28] COSTA, C. H. A.; ILHA, M. S. O. Componentes BIM de sistemas prediais hidráulicos e sanitários baseados em critérios de desempenho. Ambiente construído, Porto Alegre, v. 17, n. 2, p. 157-174, Jun 2017.

[29] COSTA, H. G. Introdução ao Método de Análise Hierárquica (Análise Multicritério no Auxílio À Decisão). 1. ed. Niterói, RJ, Brasil. v. 1. 104p, 2002.

[30] SAATY, T. L. The Analytic Hierarchy Process. N. York, USA: McGraw-Hill, 1980.

[31] NASCIMENTO, L. A.; SANTOS, E. T. A indústria da construção na era da informação. Ambiente Construído, v. 3, n. 1, p. 69-81, 2003. 